\title{
Molecular targets and novel therapeutic avenues in soft-tissue sarcoma
}

\author{
A. Elkrief $\mathrm{MD}^{* \dagger}$ and T. Alcindor $\mathrm{MD}^{*}$
}

\begin{abstract}
Soft-tissue sarcoma (STS) represents a heterogeneous group of rare tumours, and a significant number of affected patients will develop metastatic disease. Outcomes in the population with metastatic disease are generally poor, especially after progression on standard chemotherapy. The advent of personalized medicine has permitted oncologists to offer targeted treatment, thus addressing the limited treatment options and poor prognosis after progression on first-line chemotherapy. In this review, we delineate the existing data and therapeutic successes with respect to existing and emerging molecular targets in STS and options for immunotherapy in STS. Our review also summarizes emerging clinical trials that are currently recruiting patients.
\end{abstract}

Key Words Sarcoma, chemotherapy, targeted therapy, immunotherapy

Curr Oncol. 2020 February;27(S1):34-40

www.current-oncology.com

\section{INTRODUCTION}

Soft-tissue sarcoma (STS) represents a heterogeneous group of rare tumours ${ }^{1}$. Of patients with early-stage disease treated with locoregional modalities such as surgery and radiation, approximately $40 \%$ will develop metastatic disease; and of patients presenting for the first time with sTs, $10 \%$ will be diagnosed with metastatic disease $^{2}$. Goals of treatment in the metastatic setting are to palliate symptoms and improve quality of life and survival. Doxorubicin with or without ifosfamide is the firstline standard of care for the treatment of metastatic STS. Angiosarcomas are also sensitive to anthracycline-based chemotherapy $^{3}$, but taxane-based chemotherapy also has significant activity ${ }^{4-10}$.

For patients with metastatic disease, outcomes are generally poor, with overall survival ranging between 12 and 19 months, and little improvement in outcomes has been observed since the start of the $2000 \mathrm{~s}^{2}$. There is therefore an unmet clinical need to develop and implement novel treatment modalities that will improve outcomes for patients with metastatic sTS.

The advent of personalized medicine has permitted oncologists to offer targeted treatment, addressing the limited treatment options and poor prognosis after progression on first-line chemotherapy. In this review, we delineate the existing data and therapeutic successes with respect to existing and emerging molecular targets in STS. The sTSs with targetable molecular abnormalities in the adult population reviewed in detail here include desmoid tumours, tenosynovial giant-cell tumours, SMARCB1deficient sarcomas, and $T R K$ fusion sarcomas. Nextgeneration sequencing (NGS) has allowed for the identification of driver mutations and potentially targetable molecular abnormalities in patients with limited treatment options, and recent data using NGS in the sTS setting are also discussed in the review.

\section{REVIEW}

\section{Desmoid Tumours}

Desmoid tumour, also known as aggressive fibromatosis, is a rare disease of fibroblastic proliferation, characterized by mutations of the CTNNB1 (beta-catenin) or APC (familial adenomatous polyposis) gene. Although this neoplasm lacks metastatic potential, it is often locally aggressive and is characterized by infiltrative growth of adjacent tissues into the mesentery, neurovascular structures, and visceral organs $^{1}$. Patients diagnosed before the age of 40 are more likely to be women, and the most common site of onset in these patients is the abdominal wall. After the age of 40 , these tumours develop in men and women equally, and no predilection for a particular tumour site is observed in older adults. Desmoid tumours are treated initially with active surveillance ${ }^{11,12}$, because about $20 \%$ of patients can experience spontaneous regression ${ }^{13}$. Upon progression, or in the presence of life-threatening symptoms or of significant 
symptoms that cannot be controlled with pain control measures, active therapy is offered. If the patient is a candidate for surgery and the desmoid tumour is located in the abdominal wall, surgery is generally the next step. It is important to note that rates of local recurrence are high ${ }^{1}$. If the desmoid tumour arises in other sites, such as the head-and-neck area, the extremities, or the intra-abdominal or pelvic areas, systemic therapy is usually offered. Typically given for 6-12 months, common cytotoxic chemotherapy regimens includelow-dose vinblastine (or vinorelbine) and methotrexate for patients who have low burden-of-disease symptoms ${ }^{14}$, and liposomal doxorubicin for patients having more severe symptoms or experiencing progression after a prior regimen ${ }^{15}$.

\section{Targeted Therapy in Desmoid Tumours}

Imatinib: Imatinib has a limited role in patients with desmoid tumours. A phase II study in which 38 patients with unresectable desmoid tumour received imatinib reported an overall response rate of $19 \%^{16}$, but no control group was used. A retrospective case series reviewed the response rate to imatinib combined with radiation therapy in 4 patients with unresectable or recurrent desmoid tumours; all patients either experienced a partial response or had stable disease ${ }^{17}$. Given the paucity of prospective data, the use of imatinib in patients with desmoid tumours cannot be recommended at this time.

As already mentioned, desmoid tumours are more likely to be diagnosed in women before the age of 40 , often during pregnancy or within 1 year postpartum ${ }^{18}$. In support of those epidemiologic findings, immunohistochemical studies have demonstrated the presence of estrogen receptor beta in $90 \%$ of desmoid tumours ${ }^{19}$. The selective estrogen receptor modulator tamoxifen has been used in the treatment of desmoid tumours ${ }^{20,21}$, but a more recent publication showed no relationship between symptomatic benefit and the magnetic resonance imaging response with the use of tamoxifen ${ }^{22}$.

Sorafenib: Sorafenib, an oral multi-targeted receptor tyrosine kinase inhibitor, has shown activity in desmoid tumours. A retrospective analysis of 26 patients evaluated the efficacy of sorafenib in desmoid tumours that were unresectable or resectable only with amputation ${ }^{23}$. The most common primary site in the cohort was abdomen or pelvis. A significant proportion of patients (42\%) received sorafenib in the first-line setting. Sorafenib was started at $400 \mathrm{mg}$ daily and was decreased in cases of dose toxicity. As evaluated by the Response Evaluation Criteria in Solid Tumors (version 1.1), sorafenib was associated with a partial response rate of $25 \%$, with $70 \%$ of patients having stable disease. Additionally, approximately $70 \%$ of patients reported a subjective decrease in pain and use of analgesics with sorafenib, with most of those clinical improvements being noted within 2 weeks of sorafenib initiation. The study also noted that the Response Evaluation Criteria in Solid Tumors might underestimate the treatment response and suggested that the signal from T2-weighted magnetic resonance imaging might be more clinically relevant and accurate.

That retrospective trial prompted Gounder et al. ${ }^{24}$ to conduct a prospective double-blind randomized phase III trial to evaluate the safety and efficacy of sorafenib in the treatment of desmoid tumours. The study randomized 87 adult patients with either symptomatic, recurrent, or primary desmoid tumour that was deemed unresectable to receive sorafenib at a dose of $400 \mathrm{mg}$ daily or placebo. Patients who had previously received sorafenib were excluded from the trial. Crossover after progression was allowed for patients receiving placebo. The 2 -year progression-free survival rate was $81 \%$ in the sorafenib group compared with $36 \%$ in the placebo group [hazard ratio for progression or death: $0.13 ; 95 \%$ confidence interval (CI): 0.05 to $0.31 ; p<0.001$ ]. Before crossover, the objective response rate was $33 \%$ in the sorafenib group (95\% CI: $20 \%$ to $48 \%$ ) and $20 \%$ in the placebo group (95\% CI: $8 \%$ to $38 \%$ ). The most frequently reported adverse effects in the sorafenib group were grade 1 or 2 rash, fatigue, hypertension, and diarrhea. Of the patients in the sorafenib group, $29 \%$ experienced a grade 3 adverse event, and the incidences of grade 4 thrombocytopenia and anemia were each $2 \%$.

The trial demonstrated a role for sorafenib in delaying disease progression in patients with desmoid tumours, but also supported a role for observation in patients without symptoms, because $20 \%$ of the patients in the placebo group experienced spontaneous regression.

Gamma-Secretase Inhibitor PF-03084014: A phase II trial evaluated gamma-secretase inhibitor PF-03084014 (nirogacestat) in 17 patients with refractory desmoid tumours. Results were encouraging, with 5 patients (29\%) experiencing a confirmed partial response, and another 5 patients having prolonged stable disease $\mathrm{e}^{25}$. Those findings have led to a randomized placebo-controlled phase III study of nirogacestat, which is currently ongoing (see NCT03785964 at https://ClinicalTrials.gov/).

\section{Tenosynovial Giant-Cell Tumours}

Tenosynovial giant-cell tumour (TGCT), previously known as pigmented villonodular synovitis, is a rare and locally aggressive neoplasm of the joint or tendon sheath ${ }^{1}$. This neoplasm is characterized by the proliferation of synovial cells that initiate recruitment of inflammatory cells such as macrophages and histiocytes ${ }^{1}$. The tumours can be either localized or diffuse, the latter characterized by diffuse proliferation of the entire synovium, most commonly affecting the knee joint ${ }^{26}$. Although most of these neoplasms occur within the joints, a diffuse form can occur, and extraarticular forms have also been described ${ }^{27}$. Rarely, TGCT can metastasize to the adjacent lymph nodes and to the lungs ${ }^{28}$. Localized TGCT is curable with arthroscopic or open surgery, but the diffuse form of the disease is associated with a high rate of local recurrence ${ }^{29}$.

\section{Targeted Therapy in TGCT}

Pexidartinib: The neoplastic cells in giant-cell tumours can overexpress colony-stimulating factor 1 (CSF1), with signalling between CSFl and the CSFl receptor (CSF1R) ${ }^{30,31}$. Colony-stimulating factor 1 is often found on the membranes of immune cells. Given the recruitment of inflammatory cells by the neoplastic synovial cells implicated in the pathophysiology of TGCT, it was hypothesized that 
inhibition of signalling between CSFl and CSFlR could target the underlying cause of the disease.

A phase I study of 43 patients receiving pexidartinib, a dual inhibitor of CSFl $\mathrm{R}$ and the $\mathrm{c}-\mathrm{Kit}$ receptor tyrosine kinase, led to a multicentre single-cohort extension study of pexidartinib administrated at a dose of $1000 \mathrm{mg}$ daily in 23 patients with TGCT ${ }^{32}$. Patients with such tumours were included in the study if they had shown disease progression or if the tumour was recurrent or inoperable, or had required extensive surgery for complete resection. Most patients had disease within the knee. Of the 23 patients, 12 experienced a partial response, and 7 had stable disease, representing an overall response rate of 52\% (95\% CI: $32 \%$ to $73 \%$ ) and a rate of disease control (complete response, partial response, or stable disease) of $83 \%$ (95\% CI: $67 \%$ to $98 \%)$. Treatment-related adverse events occurred in $25 \%$ of the study population, and the most common adverse events included hair colour change, fatigue, nausea, dysgeusia, and periorbital edema. The most common grade 3 adverse event was elevated serum aspartate aminotransferase or alanine aminotransferase, which occurred in 3 patients.

A phase III randomized controlled trial of pexidartinib in 174 patients with TGCT was recently published in The Lan$c t^{33}$. The trial was conducted in two parts, the first being a randomized controlled trial comparing pexidartinib with placebo in patients with inoperable disease. Patients who completed the first part of the trial were eligible to enter the second part, in which they were offered open-label pexidartinib in an intention-to-treat analysis. Compared with $0 \%$ in the placebo group, $39 \%$ of the patients in the pexidartinib group achieved a response (95\% CI: $27 \%$ to $52 \% ; p<0.0001)$. In a secondary endpoint analysis, patients receiving pexidartinib, compared with those in the placebo group, reported improved range of motion and physical functioning. Of the patients in the pexidartinib group, $13 \%$ experienced serious adverse events (a toxicity profile similar to that described in the phase I study outlined earlier), with elevations in liver enzymes being a frequent and important side effect.

Taken together, the data demonstrate that pexidartinib should be considered in the treatment of individuals with TGCT and functional limitation where surgery is not possible.

Emactuzumab: A phase I study in 29 patients evaluated the use of emactuzumab (an immunoglobulin G1 subclass humanized recombinant monoclonal antibody directed against CSFlR expressed on the surfaces of macrophages) in patients with unresectable TGCT $^{34}$. Expansion cohorts for the study are currently ongoing (see NCT01494688 at https://ClinicalTrials.gov/). In the safety expansion cohort, independent central review of magnetic resonance imaging was conducted for 28 of 29 patients, and independent central review of positron-emission tomography imaging, for 26 of 29 patients. Of the 28 reviewed patients, 24 (86\%) achieved an objective response, and 19 (68\%) achieved a partial response. Of the 26 reviewed patients, 24 (92\%) achieved a metabolic response. Common adverse events after emactuzumab treatment were facial edema, fatigue, asthenia, and pruritus. Drug-induced lupus erythematous was a rare, but important, side effect.
Nilotinib: Nilotinib is a multi-targeted tyrosine kinase inhibitor that has activity against $\mathrm{CSF} \mathrm{R}^{35}$. Its efficacy was evaluated in a phase II study involving 51 patients with advanced or unresectable TGCT $^{36}$. Although a high proportion of those patients were progression-free at 12 weeks, the overall response rate was lower than had been seen with pexidartinib and emactuzumab. Nearly all patients experienced at least 1 adverse event, and $11 \%$ experienced at least 1 grade 3 adverse event (headache, hepatic enzyme abnormalities, diarrhea).

Imatinib: Imatinib has weak activity against $\operatorname{CSF} 1 \mathrm{R}^{35}$. In a retrospective series of 29 patients with locally advanced or metastatic TGCT, the objective response rate was $19 \%$, and $74 \%$ of the patients reported symptomatic or functional improvement ${ }^{37}$. Aside from that retrospective series, case reports have described success with imatinib, but the data are currently insufficient to recommend this agent in the treatment of TGCT.

\section{Tazemetostat In Ini1-Deficient Sarcomas}

Epithelioid sarcoma is a rare STS, and loss of Inil occurs in more than $90 \%$ of these tumours. Because Inil is a negative regulator of the oncogenic driver EZH2, inhibition of EZH2 was hypothesized to potentially be able to lead to tumour regression ${ }^{38}$. Tazemetostat, an inhibitor of EZH2, was evaluated in a phase II study enrolling 62 patients with advanced Inil-deficient epithelioid sarcoma. The overall response rate was $13 \%$. Additionally, 35 patients had a best response of stable disease. Those results came from an interim analysis and remain unpublished.

\section{Larotrectinib in TRK Fusion-Positive Sarcoma}

Rarely, adults with sTs have gene fusions involving NTRK. Larotrectinib, a selective NTRKinhibitor, was shown to be effective in a combined analysis of 55 patients with various NTRK fusion-positive solid tumours-an important subset of which consisted of patients with STs ${ }^{39}$. Of 11 patients with STS showing NTRK fusions, 10 experienced an objective response, and 1 achieved a complete response. No patient in the study discontinued larotrectinib because of treatment-related toxicity. The most common side effects included elevations in liver enzymes, fatigue, nausea, vomiting, dizziness, diarrhea, constipation, and cough.

\section{MET/ALK Inhibition}

Thus far, combined MET and ALK inhibition using crizotinib has been evaluated in a phase II study of 20 patients with chemotherapy-refractory alveolar rhabdomyosarcoma ${ }^{40}$. More common in children and adolescents, alveolar rhabdomyosarcoma is a rare subtype of sTs in adults, accounting for only $3 \%$ of cases. Unfortunately, the crizotinib study did not reveal any meaningful activity in the adult patient population. It is important to note that most patients in the study did not harbour an $A L K$ mutation. A second study evaluated the role of MET and ALK inhibition in 53 patients with alveolar soft-part sarcoma, another orphan disease in adults ${ }^{41}$. That study showed important clinical activity in patients with $M E T$-positive disease, most of whom had stable disease with treatment. 


\section{Emerging Immunotherapy Regimens for STS}

\section{Pembrolizumab}

Pembrolizumab, a PD-1 inhibitor, was tested in a phase II trial involving 86 patients with refractory sTs ${ }^{42}$. The primary endpoint of overall response was not met in the study, but pembrolizumab was associated with promising response rates in patients with undifferentiated pleomorphic sarcoma and dedifferentiated liposarcoma. Results of expanded cohorts for those sTs subtypes are awaited. A second phase II study, this time in multiple cohorts of patients with STS, demonstrated limited activity for pembrolizumab in combination with cyclophosphamide ${ }^{43}$. The combination of nivolumab (a PD-1 inhibitor) and ipilimumab (a CTLA-4 inhibitor) showed promising results in a phase II trial involving patients with metastatic sarcoma (not limited to STS) ${ }^{44}$.

Between $1 \%$ and $5 \%$ percent of patients with sTs have high microsatellite instability, which usually predicts response to immune checkpoint inhibitors targeting PD-1 or PD-L1 ${ }^{45}$. Although not tested specifically in patients with sTs, immune checkpoint inhibitors might reasonably be considered for patients with high microsatellite instability who progress on standard treatment.

\section{New York Esophageal Squamous Cell Carcinoma 1}

New York esophageal squamous cell carcinoma 1 (NY-ESO-1) is a marker on both T cells and B cells that can be targeted by CMB305, an active immunotherapy regimen that uses dendritic cell-targeting lentiviral vectors. A phase IB study that enrolled 25 patients with treatment-refractory STS demonstrated interesting response rates, with favourable progression-free and overall survival outcomes ${ }^{46}$.

\section{Adoptive T Cell Transfer}

Adoptive transfer of T cells engineered to express NY-ESO-1 was studied as part of an ongoing phase IB trial in patients with synovial sTs, demonstrating, in $50 \%$ of patients ( 6 of 12), antitumour responses that were characterized by tumour shrinkage over several months. In the study, correlative science demonstrated that self-regenerating pools of CD8+ NY-ESO-1 T cells produce a continuing supply of effector cells for several months ${ }^{47}$. Two other studies examining similar treatment are currently underway (see NCT01477021 and NCT01477021 at https://ClinicalTrials. gov/). Other targets for adoptive cell therapy such as MAGE-A3, MAGE-A4, and MAGE-A10 are also emerging and will be studied in clinical trials in the near future ${ }^{48}$.

\section{Chimeric Antigen Receptor T Cell Therapy}

Chimeric antigen receptors (CARs) provide another way of invigorating the immune system to kill cancer cells. In this technique, T cells are modified and equipped with CARs that redirect the antigen specificity of immune effector cells. Treatments can have important clinical activity, but sometimes cause severe adverse events by way of a cytokine storm. Use of this technique is currently being studied for many solid tumours, including a GD-2-targeting CAR in ganglioside-positive sarcomas (NCT02107963, NCT01953900) ${ }^{48}$.

\section{Vaccine-Based Therapies}

Vaccine-based therapies have been used as another method of stimulating the anticancer immune response. As mentioned earlier, the potential for using NY-ESO-1 as a vaccinebased therapy is a promising emerging option. Another vaccine trial (of $S Y T-S S X$ breakpoint peptide vaccine) that enrolled 21 patients with synovial sTs demonstrated encouraging results ${ }^{49}$.

\section{Targeted Therapies}

Approximately one third of patients with sTs have specific genetic alterations, and it is therefore important to consider targeted therapy as a potential therapeutic avenue. A large study of 584 patients that sought to characterize genomic alterations in adult patients with sTs was recently published. The authors found a median of 4 genomic alterations per patient. At least 1 targetable genomic alteration was found in 239 patients $(41 \%)^{50}$. Another large study of 5635 patients examined the role of NGS in sTs, finding meaningful clinical impacts. For example, nearly $10 \%$ of patients were found to have mutations suspicious for germline defects (including BRCA). In $16 \%$ of patients, treatment-linked alterations were found that are known to respond to a drug approved by the U.S. Food and Drug Administration, and in 7\%, alterations were found that could make the patient eligible for a clinical drug trial. Of the patients in the study, $30 \%$ were enrolled to a clinical study, and partial and complete responses were seen in patients with alterations in NTRK, IDH1, BRAF, PI3K/mTOR, and MDM2, among others. Furthermore, use of NGS altered the initial pathology diagnosis in $5 \%$ of patients enrolled to the study. In another study involving 20 patients with liposarcoma for whom NGS was used, 11 subsequently received MDM2 or CDK4 inhibition as part of a phase I clinical trial ${ }^{51}$. Meaningful clinical activity was observed in 7 patients who experienced stable disease. Although the possibilities are exciting, the incidence of actionable mutations leading to outcome-altering treatment is unknown, and given the cost of the technique, widespread use of NGS cannot be recommended at this time.

\section{SUMMARY AND FUTURE DIRECTIONS}

A significant proportion of patients with sTs present with, or develop, metastatic disease. Given the poor outcomes in STS, novel therapeutic avenues have to be developed. Many clinical trials are actively recruiting patients with advanced sts (Table I). Promising options include the use of sorafenib in desmoid tumours, pexidartinib or emactuzumab in TGCT, tazemetostat in Inil-deficient sarcomas, and larotrectinib in TRKfusion-positive sarcomas. Immunotherapy is also proving to be an emerging and promising avenue for patients with advanced sTs. Next-generation sequencing might provide insights for personalized therapy. Altogether, novel therapies in sTs are proving to be an exciting and active area of cancer research, and more data are eagerly awaited.

\section{CONFLICT OF INTEREST DISCLOSURES}

We have read and understood Current Oncology's policy on disclosing conflicts of interest, and we declare the following interests: TA 
TABLE I Clinical trials that are actively recruiting patients with advanced soft-tissue sarcoma (STS)

\begin{tabular}{|c|c|c|c|c|c|c|}
\hline \multirow{2}{*}{$\begin{array}{c}\text { ClinicalTrials.gov } \\
\text { ID }\end{array}$} & \multicolumn{2}{|l|}{ Patients } & \multirow[t]{2}{*}{ Intervention } & \multirow{2}{*}{$\begin{array}{l}\text { Primary } \\
\text { outcome }\end{array}$} & \multirow{2}{*}{$\begin{array}{l}\text { Trial } \\
\text { status }\end{array}$} & \multirow[t]{2}{*}{ Location } \\
\hline & Population & (n) & & & & \\
\hline \multicolumn{7}{|l|}{ Targeted therapy } \\
\hline NCT03104335 & $\begin{array}{l}\text { Advanced STS, } \\
\text { previously treated; } \\
\text { rhabdomyosarcoma and } \\
\text { liposarcoma excluded }\end{array}$ & 100 & Apatinib & ORR & Recruiting & $\begin{array}{c}\text { Peking University } \\
\text { International Hospital, } \\
\text { P.R.C. }\end{array}$ \\
\hline NCT03526679 & $\begin{array}{l}\text { Advanced STS, } \\
\text { previously treated; } \\
\text { leiomyosarcoma, } \\
\text { liposarcoma }\end{array}$ & 30 & Lenvatinib and eribulin & ORR & Recruiting & $\begin{array}{l}\text { National Taiwan } \\
\text { University Hospital, } \\
\text { Taiwan }\end{array}$ \\
\hline NCT02357810 & $\begin{array}{c}\text { Advanced STS, } \\
\text { previously treated; } \\
\text { liposarcoma excluded }\end{array}$ & 136 & $\begin{array}{c}\text { Pazopanib } \\
\text { hydrochloride } \\
\text { and } \\
\text { topotecan } \\
\text { hydrochloride }\end{array}$ & $\begin{array}{l}\text { Progression-free } \\
\text { rate }\end{array}$ & Recruiting & $\begin{array}{c}\text { Northwestern University, } \\
\text { IL, U.S.A. }\end{array}$ \\
\hline NCT02601950 & $\begin{array}{l}\text { Synovial sarcoma, } \\
\text { previously treated }\end{array}$ & 250 & Tazemetostat & PFS, ORR & Recruiting & Multicentre, U.S.A. \\
\hline \multicolumn{7}{|l|}{ Immunotherapy } \\
\hline NCT04052334 & $\begin{array}{l}\text { Advanced STS, } \\
\text { previously treated }\end{array}$ & 5 & $\begin{array}{l}\text { Lymphodepletion plus } \\
\text { adoptive cell therapy } \\
\text { with high-dose IL-2 }\end{array}$ & Safety & Recruiting & $\begin{array}{c}\text { H. Lee Moffitt Cancer Center } \\
\text { and Research Institute, } \\
\text { FL, U.S.A. }\end{array}$ \\
\hline NCT03056001 & $\begin{array}{l}\text { Advanced STS, } \\
\text { previously untreated }\end{array}$ & 30 & $\begin{array}{l}\text { Pembrolizumab and } \\
\text { doxorubicin }\end{array}$ & Safety & Recruiting & Multicentre \\
\hline NCT02496520 & $\begin{array}{c}\text { Metastatic or relapsed } \\
\text { sarcoma }\end{array}$ & 10 & $\begin{array}{l}\text { Dendritic cell-based } \\
\text { immunotherapy }\end{array}$ & Safety & Recruiting & $\begin{array}{l}\text { Clinica Universidad de } \\
\text { Navarra, Universidad } \\
\text { de Navarra, Spain }\end{array}$ \\
\hline NCT04095208 & $\begin{array}{l}\text { Advanced STS, } \\
\text { previously treated }\end{array}$ & 67 & $\begin{array}{l}\text { Nivolumab and } \\
\text { relatlimab }\end{array}$ & $\begin{array}{l}\text { Progression-free } \\
\text { rate }\end{array}$ & Recruiting & Institut Bergonié, France \\
\hline NCT03317457 & $\begin{array}{l}\text { Advanced STS, } \\
\text { previously untreated }\end{array}$ & 100 & $\begin{array}{l}\text { Durvalumab and } \\
\text { tremelimumab }\end{array}$ & OS & Recruiting & Multicentre \\
\hline NCT03590210 & $\begin{array}{l}\text { Advanced STS, } \\
\text { previously treated }\end{array}$ & 74 & & PFS, safety & Recruiting & $\begin{array}{l}\text { University Medicine } \\
\text { Greifswald, Germany }\end{array}$ \\
\hline
\end{tabular}

ORR = objective response rate; PFS = progression-free survival; OS = overall survival.

has received fees as an advisory board member for Merck, Eisai, Pfizer, Novartis, and Taiho; TA's institution receives funding from Karyopharm, SpringWorks, Lilly, Deciphera for trials in which he is a co-investigator. AE has no conflicts to disclose.

\section{AUTHOR AFFILIATIONS}

*Cedars Cancer Centre, Department of Oncology, McGill University Health Centre, and ${ }^{\dagger}$ Segal Cancer Centre, Department of Oncology, Sir Mortimer B. Davis Jewish General Hospital, Montreal, QC.

\section{REFERENCES}

1. Fletcher CDM, Bridge JA, Hogendoorn PCW, Mertens F, eds. WHO Classification of Tumours of Soft Tissue and Bone. 4th ed. Lyon, France: International Agency for Research on Cancer; 2013.

2. Italiano A, Mathoulin-Pelissier S, Cesne AL, et al. Trends in survival for patients with metastatic soft-tissue sarcoma. Cancer 2011;117:1049-54.

3. Young RJ, Natukunda A, Litière S, Woll PJ, Wardelmann E, van der Graaf WT. First-line anthracycline-based chemotherapy for angiosarcoma and other soft tissue sarcoma subtypes: pooled analysis of eleven European Organisation for Research and Treatment of Cancer Soft Tissue and Bone
Sarcoma Group trials. Eur J Cancer 2014;50:3178-86.

4. Skubitz KM, Haddad PA. Paclitaxel and pegylated-liposomal doxorubicin are both active in angiosarcoma. Cancer 2005; 104:361-6.

5. Fata F, O'Reilly E, Ilson D, et al. Paclitaxel in the treatment of patients with angiosarcoma of the scalp or face. Cancer 1999; 86:2034-7.

6. Nagano T, Yamada Y, Ikeda T, Kanki H, Kamo T, Nishigori C. Docetaxel: a therapeutic option in the treatment of cutaneous angiosarcoma: report of 9 patients. Cancer 2007;110:648-51.

7. Penel N, Bui BN, Bay JO, et al. Phase II trial of weekly paclitaxel for unresectable angiosarcoma: the ANGIOTAX study. J Clin Oncol 2008;26:5269-74.

8. Schlemmer M, Reichardt P, Verweij J, et al. Paclitaxel in patients with advanced angiosarcomas of soft tissue: a retrospective study of the EORTC Soft Tissue and Bone Sarcoma Group. Eur J Cancer 2008;44:2433-6.

9. Fury MG, Antonescu CR, Van Zee KJ, Brennan MF, Maki RG A 14-year retrospective review of angiosarcoma: clinical characteristics, prognostic factors, and treatment outcomes with surgery and chemotherapy. Cancer J 2005;11:241-7.

10. Young RJ, Brown NJ, Reed MW, Hughes D, Woll PJ.Angiosarcoma. Lancet Oncol 2010;11:983-91. 
11. Kasper B, Baumgarten C, Garcia J, et al. An update on the management of sporadic desmoid-type fibromatosis: a European Consensus Initiative between Sarcoma Patients EuroNet (SPAEN) and European Organization for Research and Treatment of Cancer (EORTC)/Soft Tissue and Bone Sarcoma Group (STBSG). Ann Oncol 2017;28:2399-408.

12. Fiore M, Rimareix F, Mariani L, et al. Desmoid-type fibromatosis: a front-line conservative approach to select patients for surgical treatment. Ann Surg Oncol 2009;16:2587-93.

13. Gounder MM, Thomas DM, Tap WD. Locally aggressive connective tissue tumors. J Clin Oncol 2018;36:202-9.

14. Palassini E, Frezza AM, Mariani L, et al. Long-term efficacy of methotrexate plus vinblastine/vinorelbine in a large series of patients affected by desmoid-type fibromatosis. Cancer J 2017;23:86-91.

15. Gronchi A, Jones RL. Treatment of desmoid tumors in 2019. JAMA Oncol 2019;5:567-8.

16. Kasper B, Gruenwald V, Reichardt P, et al. Imatinib induces sustained progression arrest in RECIST progressive desmoid tumours: final results of a phase II study of the German Interdisciplinary Sarcoma Group (GISG). EurJ Cancer 2017; 76:60-7.

17. Moding EJ, Million L, Avedian R, Ghanouni P, Kunder C, Ganjoo KN. Concurrent imatinib and radiation therapy for unresectable and symptomatic desmoid tumors. Sarcoma 2017;2017:2316839.

18. Fiore M, Coppola S, Cannell AJ, et al. Desmoid-type fibromatosis and pregnancy: a multi-institutional analysis of recurrence and obstetric risk. Ann Surg 2014;259:973-8.

19. Deyrup AT, Tretiakova M, Montag AG. Estrogen receptor-beta expression in extraabdominal fibromatoses: an analysis of 40 cases. Cancer 2006;106:208-13.

20. Sturt NJ, Phillips RK, Clark SK. High-dose tamoxifen and sulindac as first-line treatment for desmoid tumors. Cancer 2004;101:652.

21. Fiore M, Colombo C, Radaelli S, et al. Hormonal manipulation with toremifene in sporadic desmoid-type fibromatosis. Eur J Cancer 2015;51:2800-7.

22. Libertini M, Mitra I, van der Graaf WTA, et al. Aggressive fibromatosis response to tamoxifen: lack of correlation between MRI and symptomatic response. Clin Sarcoma Res 2018;8:13.

23. Gounder MM, Lefkowitz RA, Keohan ML, et al. Activity of sorafenib against desmoid tumor/deep fibromatosis. Clin Cancer Res 2011;17:4082-90.

24. Gounder MM, Mahoney MR, Van Tine BA, et al. Sorafenib for advanced and refractory desmoid tumors. N Engl J Med 2018;379:2417-28.

25. Kummar S, O'Sullivan Coyne G, Do KT, et al. Clinical activity of the gamma-secretase inhibitor PF-03084014 in adults with desmoid tumors (aggressive fibromatosis). JClin Oncol 2017;35:1561-9.

26. Ottaviani S, Ayral X, Dougados M, Gossec L. Pigmented villonodular synovitis: a retrospective single-center study of 122 cases and review of the literature. Semin Arthritis Rheum 2011;40:539-46.

27. Giustini N, Bernthal NM, Bukata SV, Singh AS. Tenosynovial giant cell tumor: case report of a patient effectively treated with pexidartinib (PLX3397) and review of the literature. Clin Sarcoma Res 2018;8:14.

28. Asano N, Yoshida A, Kobayashi E, Yamaguchi T, Kawai A. Multiple metastases from histologically benign intraarticular diffuse-type tenosynovial giant cell tumor: a case report. Hum Pathol 2014;45:2355-8.

29. Staals EL, Ferrari S, Donati DM, Palmerini E. Diffuse-type tenosynovial giant cell tumour: current treatment concepts and future perspectives. Eur J Cancer 2016;63:34-40.
30. Molena B, Sfriso P, Oliviero F, et al. Synovial colony-stimulating factor-1 mRNA expression in diffuse pigmented villonodular synovitis. Clin Exp Rheumatol 2011;29:547-50.

31. West RB, Rubin BP, Miller MA, et al. Alandscape effect in tenosynovial giant-cell tumor from activation of CSFl expression by a translocation in a minority of tumor cells. Proc Natl Acad Sci U S A 2006;103:690-5.

32. Tap WD, Wainberg ZA, Anthony SP, et al. Structure-guided blockade of CSFlR kinase in tenosynovial giant-cell tumor. $N$ Engl J Med 2015;373:428-37.

33. Tap WD, Gelderblom H, Palmerini E, et al. Pexidartinib versus placebo for advanced tenosynovial giant cell tumour (ENLIVEN): a randomised phase 3 trial. Lancet 2019;394:478-87.

34. Cassier PA, Italiano A, Gomez-Roca CA, et al. CSFlR inhibition with emactuzumab in locally advanced diffuse-type tenosynovial giant cell tumours of the soft tissue: a dose-escalation and dose-expansion phase 1 study. Lancet Oncol 2015;16: 949-56.

35. Brahmi M, Vinceneux A, Cassier PA. Current systemic treatment options for tenosynovial giant cell tumor/pigmented villonodular synovitis: targeting the CSF1/CSF1R axis. Curr Treat Options Oncol 2016;17:10.

36. Gelderblom H, Cropet C, Chevreau C, et al. Nilotinib in locally advanced pigmented villonodular synovitis: a multicentre, open-label, single-arm, phase 2 trial. Lancet Oncol 2018;19:639-48.

37. Ries CH, Cannarile MA, Hoves S, et al. Targeting tumorassociated macrophages with anti-CSF-1R antibody reveals a strategy for cancer therapy. Cancer Cell 2014;25:846-59.

38. Gounder M, Schöffski P, Villalobos V, et al. A phase II, multicenter study of the EZH2 inhibitor tazemetostat in adults: epithelioid sarcoma cohort (NCT02601950) [abstract 1615PD]. Ann Oncol 2018;29(suppl 8):.

39. Drilon A, Laetsch TW, Kummar S, et al. Efficacy of larotrectinib in TRK fusion-positive cancers in adults and children. NEngl J Med 2018;78:731-9.

40. Schoffski P, Wozniak A, Leahy MG, et al. The tyrosine kinase inhibitor crizotinib does not have clinically meaningful activity in heavily pre-treated patients with advanced alveolar rhabdomyosarcoma with FOXO rearrangement: European Organisation for Research and Treatment of Cancer phase 2 trial 90101 “CREATE." EUr J Cancer 2018;94:156-67.

41. Schoffski P, Wozniak A, Kasper B, et al. Activity and safety of crizotinib in patients with alveolar soft part sarcoma with rearrangement of TFE3: European Organization for Research and Treatment of Cancer (EORTC) phase II trial 90101 "CREATE." Ann Oncol 2018;29:758-65.

42. Tawbi HA, Burgess M, Bolejack V, et al. Pembrolizumab in advanced soft-tissue sarcoma and bone sarcoma (SARC028): a multicentre, two-cohort, single-arm, open-label, phase 2 trial. Lancet Oncol 2017;18:1493-501.

43. Toulmonde M, Penel N, Adam J, et al. Use of PD-1 targeting, macrophage infiltration, and IDO pathway activation in sarcomas: a phase 2 clinical trial. JAMA Oncol 2018;4:93-7.

44. D'Angelo SP, Mahoney MR, Van Tine BA, et al. Nivolumab with or without ipilimumab treatment for metastatic sarcoma (Alliance A091401): two open-label, non-comparative, randomised, phase 2 trials. Lancet Oncol 2018;19:416-26.

45. Bonneville R, Krook MA, Kautto EA, et al. Landscape of microsatellite instability across 39 cancer types. JCO Precis Oncol 2017;2017:

46. Chawla SP, Pollack S, Block M, et al. Immune response, safety, and overall survival of NY-ESO-1+ soft tissue sarcoma patients treated with CMB305 therapy [abstract 1607PD]. Ann Oncol 2018;29 (suppl 8):. [Available online at: https://academic.oup. com/annonc/article/29/suppl_8/mdy299.006/5140242; cited 6 January 2020] 
47. D’Angelo SP, Melchiori L, Merchant MS, et al. Antitumour activity associated with prolonged persistence of adoptively transferred NY-ESO-1 c259 T cells in synovial sarcoma. Cancer Discov 2018;8:944-57.

48. Lee A, Huang P, DeMatteo RP, Pollack SM. Immunotherapy for soft tissue sarcoma: tomorrow is only a day away. Am Soc Clin Oncol Educ Book 2016;35:281-90.

49. Kawaguchi S, Tsukahara T, Ida K, et al. SYT-SSX breakpoint peptide vaccines in patients with synovial sarcoma: a study from the Japanese Musculoskeletal Oncology Group. Cancer Sci 2012;103:1625-30.

50. Lucchesi C, Khalifa E, Laizet $\mathrm{Y}$, et al. Targetable alterations in adult patients with soft-tissue sarcomas: insights for personalized therapy. JAMA Oncol 2018;4:1398-404.

51. Somaiah N, Beird HC, Barbo A, et al. Targeted next generation sequencing of well-differentiated/dedifferentiated liposarcoma reveals novel gene amplifications and mutations. Oncotarget 2018;9:19891-9. 